\title{
The 3D morphology of the ejecta surrounding VY Canis Majoris
}

\author{
Terry Jay Jones ${ }^{1}$, Roberta M. Humphreys ${ }^{1}$ and L. Andrew Helton ${ }^{1}$ \\ ${ }^{1}$ Department of Astronomy, University of Minnesota, Minneapolis, MN 55455, USA \\ email: tjj@astro.umn.edu,roberta@aps.umn.edu, ahelton@astro.umn.edu
}

\begin{abstract}
We use second epoch images taken with WFPC2 on the HST and imaging polarimetry taken with the HST/ACS/HRC to explore the three dimensional structure of the circumstellar dust distribution around the red supergiant VY Canis Majoris. Transverse motions, combined with radial velocities, provide a picture of the kinematics of the ejecta, including the total space motions. The fractional polarization and photometric colors provide an independent method of locating the physical position of the dust along the line-of-sight. Most of the individual arc-like features and clumps seen in the intensity image are also features in the fractional polarization map, and must be distinct geometric objects. The location of these features in the ejecta of VY CMa using kinematics and polarimetry agree well with each other, and strongly suggest they are the result of relatively massive ejections, probably associated with magnetic fields.
\end{abstract}

Keywords. stars: individual (VY CMa), stars: mass loss, polarization

\section{Introduction}

VY CMa is one of the most luminous evolved stars known, and is near the empirical upper limit for cool hypergiants (Humphreys \& Davidson 1994, de Jager 1998). At a distance of $1.5 \mathrm{kpc}$ (Herbig 1972, Lada \& Reed 1978, Marvel 1997), the star has a luminosity of $\sim 4.3 \times 10^{5} \mathrm{~L}_{o}$. VY CMa is surrounded by an asymmetric nebula, 10" across, created by a very high mass loss rate of $4 \times 10^{-4} M_{o} y r^{-1}$ (Danchi et al. 1994). This high luminosity and high mass loss rate make VY CMa one of the richest stellar maser sources known. Multi-wavelength HST/WFPC2 images of VY CMa (Smith et al. 2001) revealed a complex circumstellar environment dominated by multiple filamentary arcs and knots. The apparent random orientations of the arcs suggested that they were produced by localized ejections, not necessarily aligned with either the stars presumed NE/SW axis (Morris \& Bowers 1980, Bowers et al. 1983, Richards et al. 1998) or its equator. Smith et al. (2001), Smith (2004), and Humphreys et al. (2005) argue that the circumstellar environment of VY CMa is dominated by complex, distinct arcs and features that were created at different epochs. To further investigate the distribution of material in the ejecta surrounding VY CMa, we use second epoch images taken with WFPC2 on the HST and imaging polarimetry taken with the HST/ACS/HRC.

\section{Kinematics}

Humphreys et al. (2007) combined previous radial velocity measurements with new multi-epoch HST images to determine the total space motion of many of the knots and arcs seen in the nebula surrounding VY CMa. They find that these features are moving at different velocities, in different directions, and at different angles relative to the plane of the sky and to the star, confirming their origin from eruptions at different times and from physically separate regions on the star. The projected motions on the sky of these 
features are illustrated in Figure 1. Most of these vectors point back to the star, indicative of purely radial motions. Under the assumption their total space motion is purely radial from the star, the addition of the radial velocity observations allows us to locate the features in 3 dimensions around the star.

The principle features and the travel time since their ejection are illustrated in Figure 2. The NW Arc is redshifted with respect to the systemic stellar velocity and stretches out behind the star. Arcs 1 and 2 are blueshifted and travelling towards us relative to the star. The S Arc has two velocity components, one of which shows a redshift, placing the arc behind the star moving away from us.

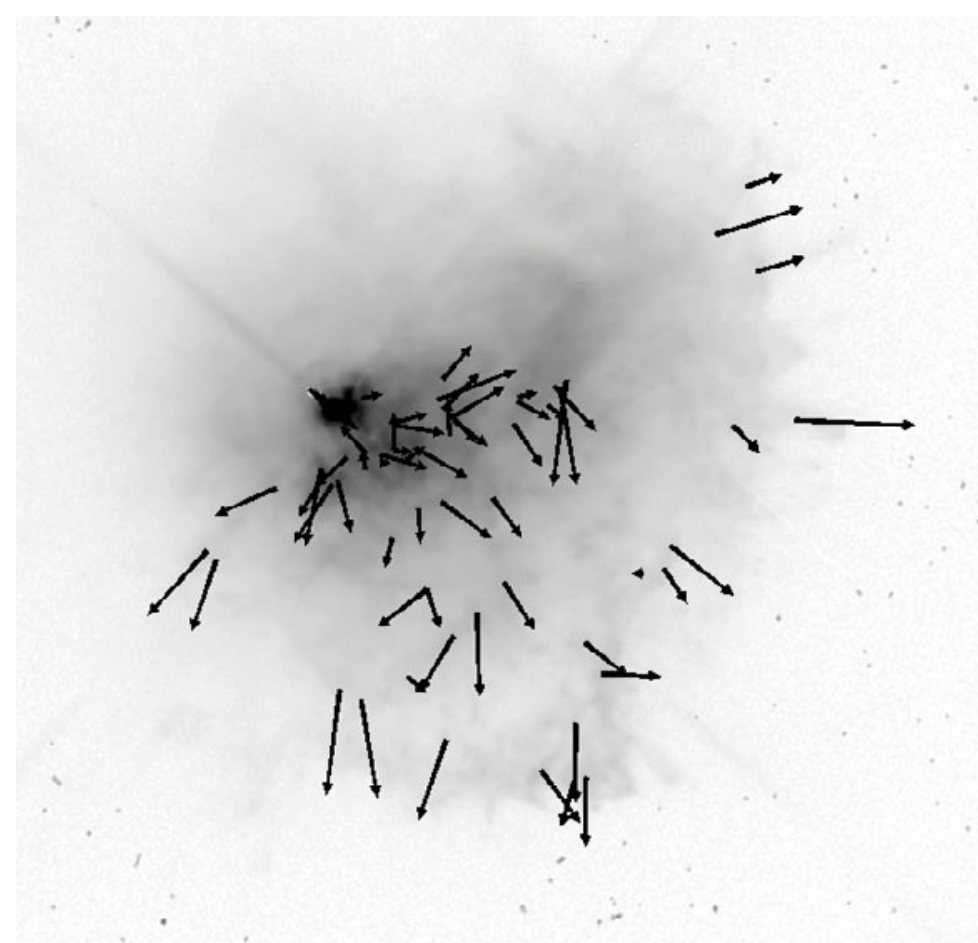

Figure 1. Proper motion vectors for features seen in the F550 image of VY CMa

\section{Polarimetry}

Polarimetry has proved a powerful tool for studying the geometry of extended circumstellar dust and gas, in particular bipolar systems. For example, Shure et al. (1995) were able to accurately fix the tilt of the bipolar outflow axis in the peculiar red giant $\mathrm{OH}$ 0739. Schulte-Ladbeck et al. (1999) were able to distinguish between different axisymmetric models for the ejecta surrounding Eta Carina. Polarimetry combined with theoretical modeling has been used to further refine the details of bipolar outflows and circumstellar disks (Lowe \& Gledhill 2007, Hoffman et al. 1997). In the visual images, VY CMa does not appear to have a mass loss nebula with any simple, underlying symmetry like a classic bipolar nebula and the analysis of the radial velocities and proper motions found a more complex distribution of ejecta. Imaging polarimetry can be used as an independent method to locate each of these features in three dimensions with respect to the star, and without reference to any model distribution.

If the scattering is optically thin, we can compute two possible scattering angles from 


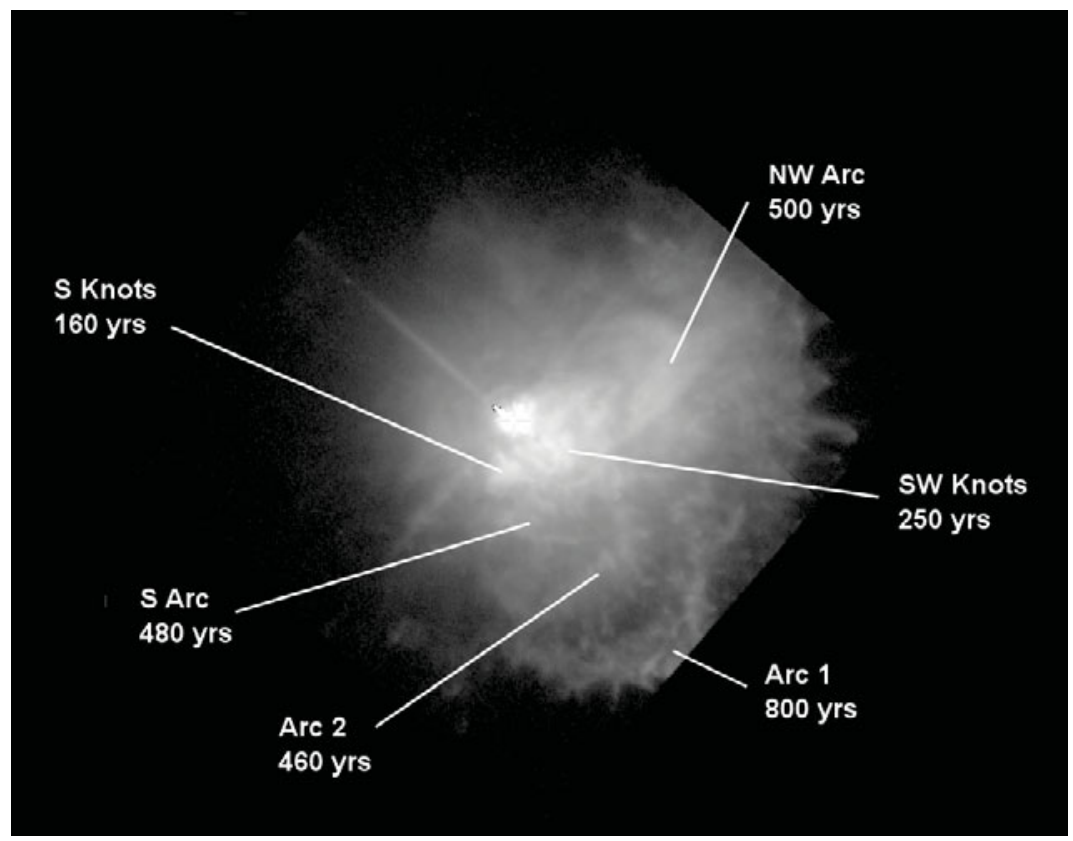

Figure 2. Principle features and an estimate of their age since ejection.

the observed fractional polarization, but we can not determine from the polarization alone whether the dust is at the larger or smaller scattering angle. For fractional polarizations less than the observed maximum, the dust must be either behind or in front of the plane of the sky containing the scattering angle corresponding to maximum polarization. In principle, light scattered off of dust behind the plane of optimum scattering will experience more reddening exiting the nebula than light scattered off of dust in front of that plane. Jones et al. (2007) used the color of the nebulosity as a means of breaking the foreground/background degeneracy in locating the dust. This technique can not be expected to extract fine details of the geometry of the dust surrounding VY CMa, only large scale patterns. Any single line of sight through the nebulosity likely intersects dust at a range of scattering angles. Given these limitations, we will concentrate on the bright, distinct regions identified in Figure 2 that are not too close to the star and too optically thick for our technique to work.

A map of fractional polarization along with a total intensity image and a map of the instrumental [547]-[1024] color from Smith et al. (2001) is shown in Figure 3. The NW Arc is prominent in the total intensity image and is quite red in the color map. In the fractional polarization map this feature is clearly apparent as more weakly polarized than its surroundings. Arcs 1 and 2 are relatively blue in color and can be seen as moderately polarized features in the fractional polarization map. The highest fractional polarization, reaching $80 \%$, is found in the regions surrounding the NW Arc. The entire NE half of the nebula is faint and has relatively low polarization, varying between $10 \%$ and $20 \%$ on average.

Most of the individual arc-like features and clumps appear to be separate geometric objects, and do not display any clear association with a simple bipolar outflow geometry. They are distinct both in the fractional polarization map and the intensity image. If these features were just local density enhancements, the fractional polarization would 


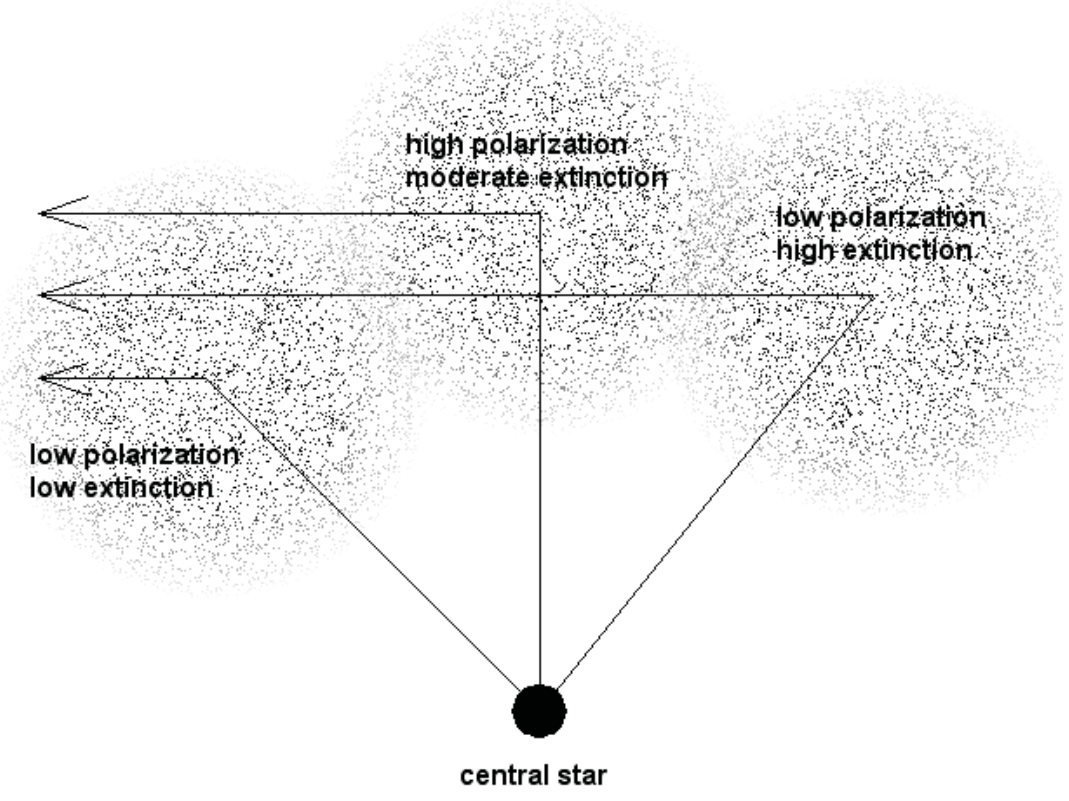

Figure 3. Schematic of the technique to use fractional polarization and color to locate a region of scattering along the line of sight.
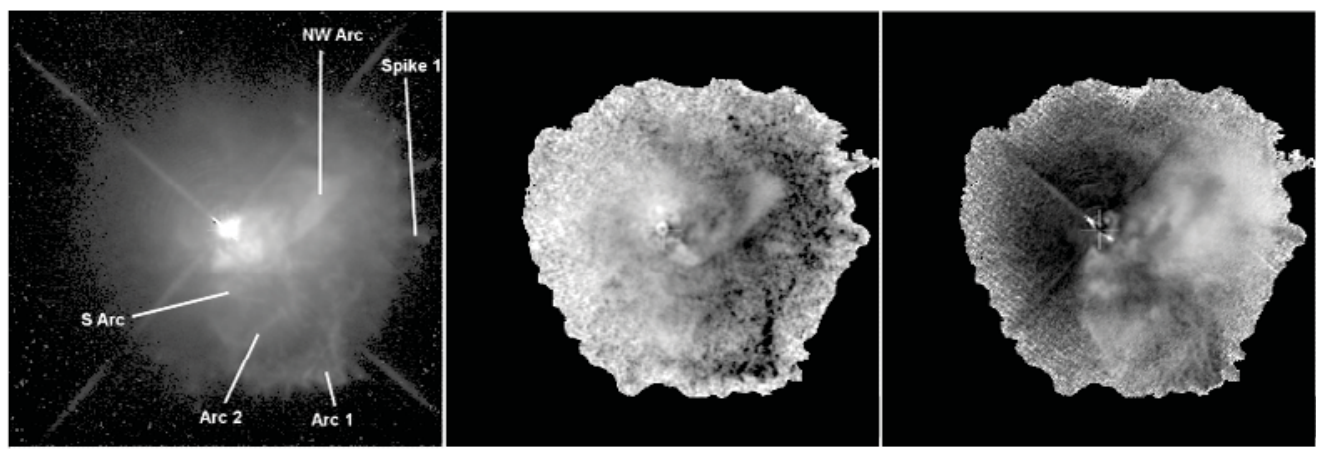

Figure 4. Left: F658 total intensity; Center: [547]-[1024] color; Right: fractional polarization.

not change across strong brightness gradients. In all cases, the locations of these features are consistent with their observed total space motions.

The polarimetry analysis places the NW Arc (which is moderately optically thick in scattering at $658 \mathrm{~nm}$ ) behind the plane of the sky at an angle with respect to the star of about $30^{\circ}$, in good agreement with the kinematic location from Humphreys et al. (2007) for features A, D, X, Y in the NW Arc. Arc 1 is in front of the star at an angle of about $30^{\circ}$ based on the polarimetry, in good agreement with the value of $35^{\circ}$ from the kinematics. Arc 2 is also in front of the star at about $20^{\circ}$ according to the polarimetry and the same value based on the kinematics. Humphreys et al. (2007) list the S Arc as being located in front of the star, but there are two velocities associated with the S Arc. The redshifted feature would place the S Arc behind the star at an orientation similar to that derived from the polarimetry. 


\section{Summary}

The morphology of the ejecta surrounding VY CMa is more complex than a simple outflow with one axis of symmetry. Most of the individual arc-like features and clumps seen in the HST images appear to be separate geometric objects, and do not display any clear association with a bipolar outflow geometry. They are distinct both in the fractional polarization map and the intensity image. If these features were just local density enhancements, the fractional polarization would not change across strong brightness gradients. Placement of these arcs and knots within the volume surrounding the central star using the observed fractional polarization and colors of the reflection nebulosity is in good agreement with the kinematics, assuming the motions are primarily radial out from the star.

Extrapolation of the space motions of these features back to the star yields different ejection times spanning the last 1000 years or so. The motions are not consistent with a single ejection time or a smooth, continuous outflow. In analogy to the Sun, we suggest these arcs and knots may be associated with very large scale magnetic phenomena at the surface of the star, but on much larger energy and mass scales.

\section{References}

Bowers, P. F., Johnston, K. J., \& Spencer, J. H. 1983, ApJ, 274, 733

Danchi, W. C., Bester, M., Degiacomi, C. G., Greenhill, L. J., \& Townes, C. H. 1994, AJ 107, 1469

de Jager, C. $1998, A \& A R$ 8, 145

Herbig, G. H. 1972, ApJ, 172, 375

Hoffman, J. L., Whitney, B. A., Wood, K., \& Nordsieck, K. H. 1997, Bulletin of the American Astronomical Society, 29, 1279

Humphreys, R. M., \& Davidson, K. 1994, PASP 106, 1025

Humphreys, R. M., Helton, L. A., \& Jones, T. J. 2007, AJ, in press.

Humphreys, R. M., Davidson, K., Ruch, G., \& Wallerstein, G. 2005, AJ, 129, 492

Jones, T. J., Humphreys, R. M., Helton, L. A., Gui, C. \& Xiang, H. 2007, AJ, in press.

Lada, C. J., \& Reid, M. J. 1978, ApJ, 219, 95

Lowe, K. T. E., \& Gledhill, T. M. 2007, MNRAS, 374, 176

Marvel, K. 1997, PASP, 109, 1286

Morris, M., \& Bowers, P. F. 1980, AJ, 85, 724

Richards, A. M. S., Yates, J. A., \& Cohen, R. J. 1998, MNRAS, 299, 319

Schulte-Ladbeck, R. E., Pasquali, A., Clampin, M., Nota, A., Hillier, D. J., \& Lupie, O. L. 1999, AJ, 118, 1320

Shure, M., Sellgren, K., Jones, T. J., \& Klebe, D. 1995, AJ, 109, 721

Smith, N. 2004, MNRAS, 349, L31

Smith, N., Humphreys, R. M., Davidson, K., Gehrz, R. D., Schuster, M. T., \& Krautter, J. 2001, AJ 121,1111 